\title{
Flexible Broadband Terahertz Modulation Based on Strain-Sensitive MXene Material
}

\author{
Yangqi Liu, Xiang Li, Tingting Yang, Jingyu Liu, Bin Liu, Jingling Shen, Bo Zhang* and \\ Fuhe Wang *
}

Key Laboratory of Terahertz Optoelectronics, Ministry of Education, Advanced Innovation Center for Imaging Technology, Beijing Key Laboratory for Terahertz Spectroscopy and Imaging, Beijing Key Laboratory of Metamaterials and Devices, Department of Physics, Capital Normal University, Beijing, China

A flexible broadband terahertz modulator based on a strain-sensitive MXene material is reported. MXene is shown to have high terahertz wave absorption through experimental testing of various substrate samples. Results show that the $\mathrm{THz}$ signal transmission increases with increasing stretching degree, which differs obviously from transmission

OPEN ACCESS

Edited by:

Yingxin Wang,

Tsinghua University, China

Reviewed by:

Zhen Tian,

Tianjin University, China

Faustino Wahaia,

Pontificia Universidad Católica de chile, Chile

Qiwu Shi,

Sichuan University, China

*Correspondence: Bo Zhang bzhang@cnu.edu.cn Fuhe Wang

wfh-phy@cnu.edu.cn

Specialty section:

This article was submitted to Optics and Photonics,

a section of the journal

Frontiers in Physics

Received: 22 February 2021

Accepted: 12 May 2021

Published: 28 May 2021

Citation:

Liu Y, LiX, Yang T, Liu J, Liu B, Shen J,

Zhang $B$ and Wang $F$ (2021) Flexible Broadband Terahertz Modulation

Based on Strain-Sensitive

MXene Material.

Front. Phys. 9:670972.

doi: 10.3389/fphy.2021.670972 through pure PVAc substrates. Analysis of the terahertz time-domain spectrum and electrical characterization indicate that the sample's conductivity decreases with increasing stretching degree. The trend and magnitude of the electrical conductivity results are also very similar to those from the time-domain spectrum. MXene is shown to be a simple, efficient terahertz broadband spectrum modulator with transmittance that can be affected by applying external forces.

Keywords: terahertz modulation, MXene, strain-sensitive, flexible, broadband

\section{INTRODUCTION}

As a unique radiation wave type, terahertz waves have had a profound impact on fields including molecular biology, medical imaging, security detection and future communication systems [1-5]. The terahertz wave modulator is the core component of terahertz wireless communications, highspeed data transmission and high-resolution imaging systems. This modulator has characteristics that include low energy requirements, low transients, broadband performance, high coherence and strong perspective properties. In recent years, increasing numbers of different terahertz modulator types have been proposed. The functions of terahertz devices (including modulators [6-11], filters [12], polarizers [13,14] and metamaterials [15-19]) are critical to the acceleration of spectral, imaging and communications applications using terahertz technology. In recent years, various methods for fabrication of high-efficiency $\mathrm{THz}$ modulators have been proposed, including fabrication of an in-band transition using graphene-induced perturbation, phase changes using vanadium dioxide $\left(\mathrm{VO}_{2}\right)$, formation of composite metamaterials using split ring resonators and silicon light excitation. Although considerable efforts have been made to design and optimize the $\mathrm{THz}$ wave dynamic tuning materials, these efforts have been hindered by the low tuning efficiency of these materials. Therefore, the efforts to develop new terahertz modulation materials with high efficiency are of major significance. In recent years, flexible optoelectronic devices have attracted widespread attention because they can operate under various mechanical deformation conditions to meet the requirements of wearable or flexible applications [20-24]. For example, in 2018, Zhang et al. reported an engineering three-dimensional integrated framework for grinding stretchable electronic products by combining material design with advanced micromachining strategies [20]. At the same 
time, some research on flexible terahertz devices was proposed [25-27], but most of the research focused on the integration of device units with flexible substrates. For example, Suzuki et al. designed terahertz metal cells that contained carbon nanotubes (CNTs) to form a flexible terahertz scanner and demonstrated the potential of this scanner in imaging of three-dimensional curvature objects [26]. Cong et al. fabricated a $\mathrm{THz}$ metamaterial quarter-wave plate composed of a metal wire grid layer and an isotropic polyimide layer that can be bent to cover a detector and used to explore the chirality of biomacromolecules [27]. However, these functions are essentially dependent on the nature of the device unit and must be performed using metamaterials with complex structures. In 2020, Shi et al. developed a flexible terahertz modulation method based on conductive polymer composites composed of thermoplastic polyurethane (TPU) and conductive $\mathrm{Ni}$ particles. This flexible layer exhibits the resistivity change of six to seven orders under tensile strain, which can be used to control the $\mathrm{THz}$ transmission with a giant modulation depth as high as $96 \%$. Starting from the structural role of metamaterials, $\mathrm{THz}$ radiation can be modulated flexibly using the properties of materials [28]. Therefore, flexible material modulators with simple structures and obvious effects are desirable.

Mxenes represent a new family of two-dimensional transition metal carbides and nitrides with the general formula $M_{n+1} X_{n} T_{x}$ [where $\mathrm{M}$ is an early transition metal ( $\mathrm{Sc}, \mathrm{Ti}, \mathrm{Zr}, \mathrm{Hf}, \mathrm{V}, \mathrm{Nb}, \mathrm{T}$ a, $\mathrm{Cr}, \mathrm{Mo}$, etc.), $\mathrm{X}$ is carbon or nitrogen, and $\mathrm{T}_{\mathrm{x}}$ represents surface terminations, (e.g. hydroxyl, oxygen, or fluorine terminations)] [38, 39]. Mxenes have attracted major interest because of their interesting and diverse mechanical, structural, physical and chemical properties. Most importantly, MXenes exhibit ultra-high conductivity (1500 S/ $\mathrm{cm}$ ) on hydrophilic surfaces [29]. Because of this unique characteristic, MXenes have attracted extensive attention in fields such as energy storage [30-32], selective ion screening [33, 34], intelligent materials [35], electromagnetic interference shielding [36], and lasers [37]. Current research indicates that MXenes show excellent responses to electromagnetic waves. Jhon reported that $\mathrm{Ti}_{3} \mathrm{C}_{2}$ has high absorption and extinction coefficients in the terahertz range based on a first principles study [38]. This type of material with its strong absorption properties has drawn our interest strongly and we have previously fabricated a flexible broadband terahertz modulator based on a strain-sensitive MXene material.

In this work, the terahertz signal absorption of an MXene $\left[\mathrm{Ti}_{3} \mathrm{C}_{2} \mathrm{~T}_{\mathrm{x}}\left(\mathrm{T}_{\mathrm{x}}=-\mathrm{OH},-\mathrm{O}\right)\right] /$ PVAc(latex) sample is obviously reduced under the application of external tension, and the terahertz wave is thus modulated effectively to a large extent. During the material stretching process, the conductivity of the sample decreases as the degree of stretching increases, which results in increased $\mathrm{THz}$ wave transmission. The $\mathrm{THz}$ modulator introduced in this paper has advantages that include a simple structure, simple implementation and an obvious effect.

\section{EXPEROMENTAL DETAILS}

The substrate was fixed on a clean quartz wafer, then a thin MXene dispersion layer was spin coated on a clean emulsion substrate $(1.5 \mathrm{~cm} \times 3 \mathrm{~cm} \times 0.2 \mathrm{~mm})$, and sintered at $100^{\circ} \mathrm{C}$ for $15 \mathrm{~min}$. Subsequently, silver parallel electrodes $(1 \mathrm{~cm}$ long $\times$ $0.5 \mathrm{~cm}$ wide $\times 200 \mathrm{~nm}$ thick, with a distance between the electrodes of $5 \mathrm{~mm}$ ) are deposited on the MXene layer by thermal evaporation in a vacuum chamber. Finally, a silver glue layer is coated onto the silver electrode and sintered at $100^{\circ} \mathrm{C}$ for $15 \mathrm{~min}$. The sample is then removed from the quartz sheet and fixed onto a stretchable sample holder.

By performing scanning electron microscopy (SEM) surface morphology tests, we obtained the surface morphologies of the samples in the relaxed and flat state and the tensile state. The SEM surface topography of the MXene $\left[\mathrm{Ti}_{3} \mathrm{C}_{2} \mathrm{~T}_{\mathrm{x}}\left(\mathrm{T}_{\mathrm{x}}=-\mathrm{OH},-\mathrm{O}\right)\right] /$ PVAc(latex) sample are shown in Figures 1B,C. Figure 1C shows the morphology of the surface and section of MXene sample in the non-tensile state (the small picture in the upper right corner is a sectional view). Figure 1C shows the surface morphology of mxene under tension. As shown in Figures 1B,C, when the magnification is 35,000 times, we can see that the surface under tension is less wrinkled and much smoother than that in the relaxed state.

A GaAs photoconductive antenna was used to generate terahertz pulses with bandwidths in the $0.2-1.2 \mathrm{THz}$ range. To detect the transmitted terahertz signals, a $\mathrm{ZnTe}$ crystal was used with an electro-optical sampling technique. The electro-optic effect is basically a nonlinear coupling effect between a lowfrequency electric field (the terahertz pulse) and an optical pulse in the sensor crystal (ZnTe). The modulation of the polarization ellipticity of an optical probe beam that passes through the $\mathrm{ZnTe}$ crystal caused by modulation of the ZnTe crystal's birefringence by a terahertz pulse is analyzed to gain information on both the amplitude and the phase of the terahertz wave. The tensile sample holder with the sample is placed into a $\mathrm{THz}$ time-domain spectroscopy (THz-TDS) system and the terahertz light spot is aimed at the titanium carbide part of the holder between the electrodes, as shown in Figure 1A. By adjusting the knob on the left side of the sample holder to adjust the stretching degree of the sample, the terahertz time-domain spectra of terahertz transmission characteristics of different stretched samples are obtained. The corresponding frequency spectrum can be obtained by applying a Fourier transform, and the conductivities of the samples with different tensile degrees can be calculated using the method described in Formula 2. The reference value is taken from the tensile data of an uncoated titanium carbide substrate.

The procedure is described as follows. First, connect the two ends of the sample via the electrodes to the source meter, and take the relaxed and flat state of the sample as the initial state; stretch the sample by a stretching amount of $5.7 \%$, which acts as the change unit, and apply voltagesranging from $1 \mathrm{~V}$ to $-1 \mathrm{~V}$; collect the current information at 10 points, obtain the current-voltage (I-V) diagram for different stretching degrees, and then calculate the resistance value and conductivity of the sample.

\section{RESULTS AND DISCUSSION}

The terahertz time domain spectra of PVAc samples with and without MXene coatings are shown in Figure 2A. The sample 


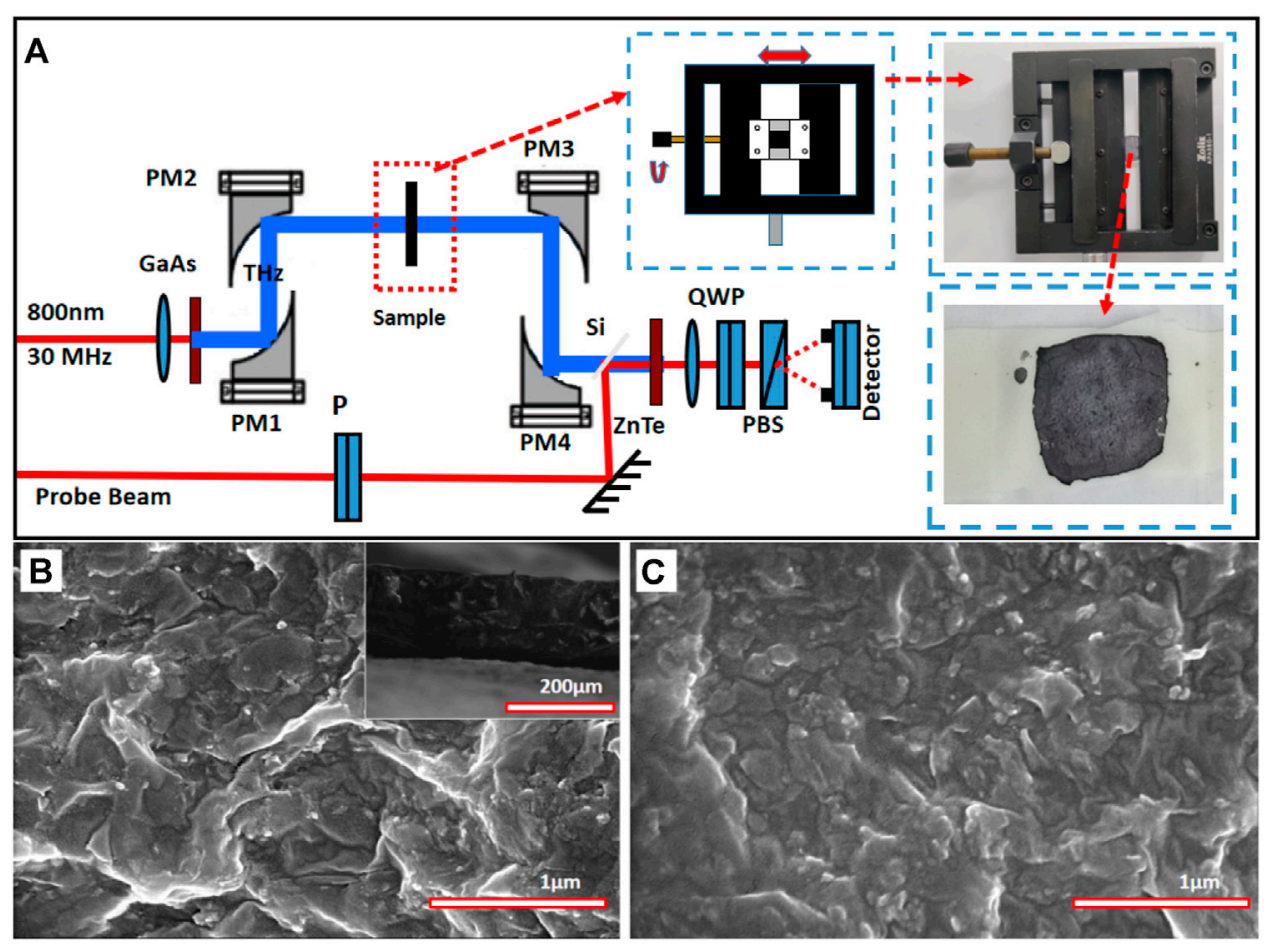

FIGURE 1 | (A) Schematic of the experimental setup. (B) Surface morphology of the sample without tension. (C) Surface morphology of the sample under tension.
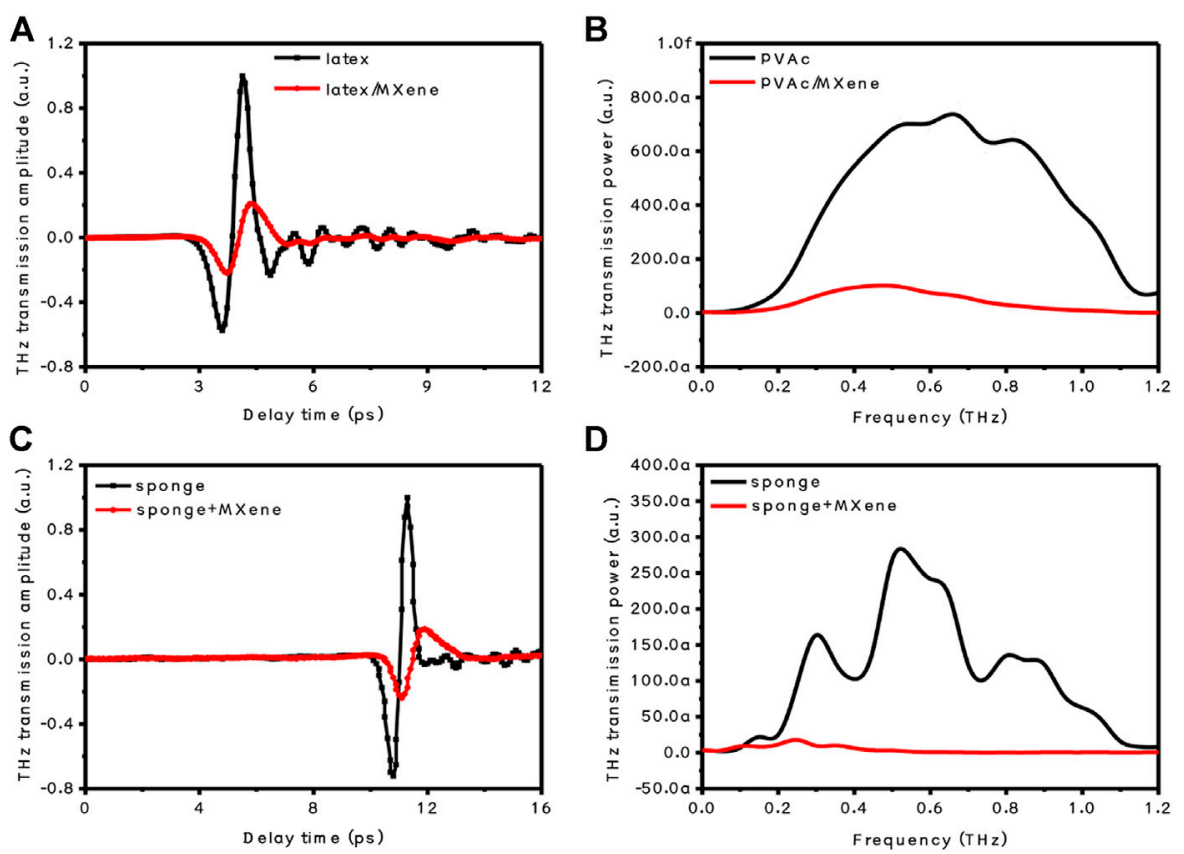

FIGURE 2 | (A) Terahertz time-domain spectra of samples with and without MXene coating on PVAc substrates. (B) Terahertz frequency-domain spectra of samples with and without MXene coating on PVAc substrates. (C) Terahertz time-domain spectra of samples with and without MXene coating on sponge substrates. (D) Terahertz frequency-domain spectra of samples with and without MXene coating on sponge substrate. 

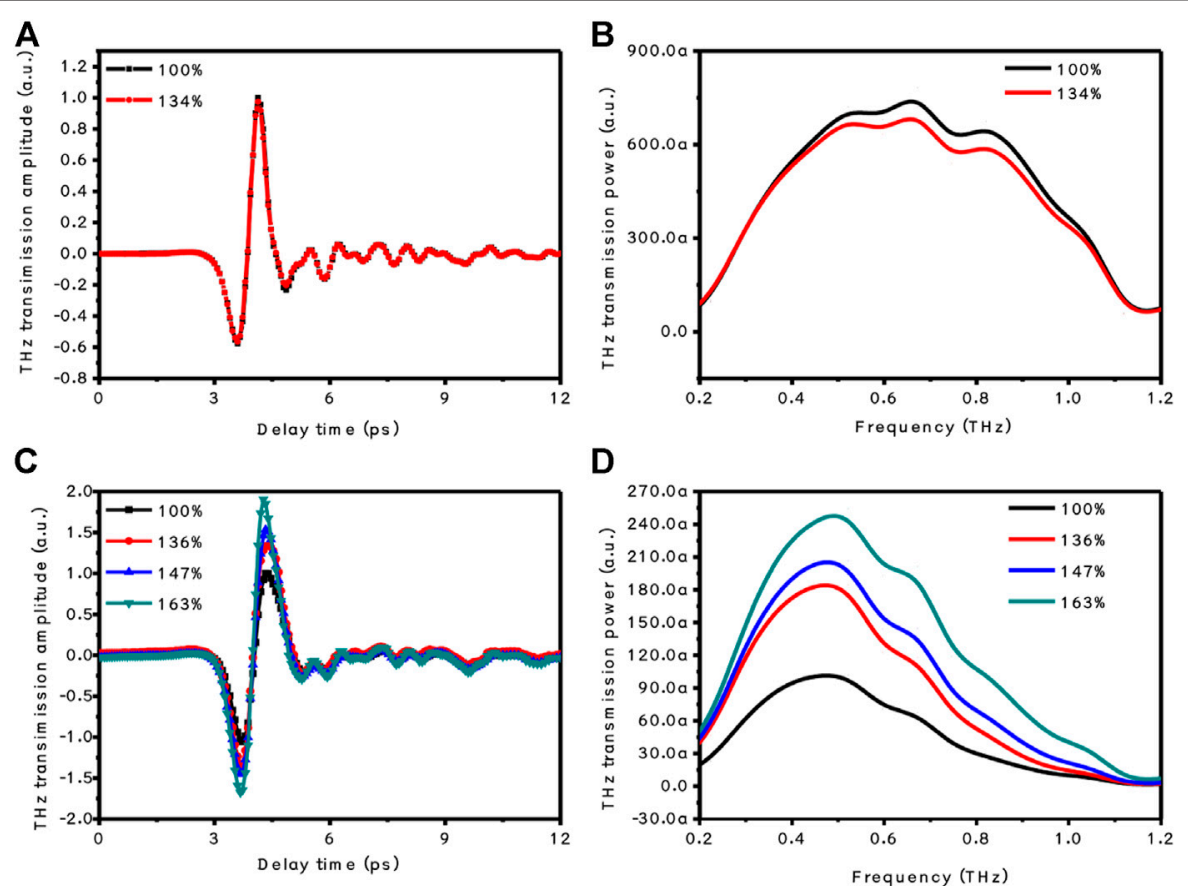

FIGURE 3 | (A) Terahertz time-domain spectra of samples without MXene coating on a PVAc substrate under tensile and non-tensile conditions. (B) Terahertz time-domain spectra of samples coated with MXene on PVAc substrate under different tensile degrees. (C) Terahertz frequency-domain spectra of samples without MXene coating on PVAc substrate under tensile and non-tensile conditions. (D) Terahertz frequency-domain spectra of samples coated with MXene on PVAc substrate under different tensile degrees.

that was coated with MXene reduced the transmission peak of the $\mathrm{THz}$ wave by approximately $80 \%$. As shown in Figure 2B, the transmission frequency spectrum of the terahertz wave shows a major transmission difference before and after spin coating of the MXene material. This shows that the MXene has a very high absorbing capacity. At the same time, a sponge material was also used as a sample substrate in an additional experiment, with results as shown in Figures 2C,D. The results obtained are similar to those for the PVAc substrate.

The tensile results for the PVAc substrate are shown in Figure 3A. These results show that the substrate selfstretching has little effect on the $\mathrm{THz}$ transmission. Figure 3B shows the terahertz transmission power spectrum of the PVAc under tension. When the substrate is stretched, the $\mathrm{THz}$ signal decreases slightly. The time-domain spectra of the MXene/ PVAc(latex) structure are studied under different tensile degrees, with results as shown in Figure 3C. All the tensile percentages in this paper are calculated by the ratio of the length after stretching to the original length. When the degree of stretching is $136 \%$, the transmission amplitude of the timedomain spectrum increases by more than $42 \%$. When the tensile strength is $147 \%$, the transmission amplitude then increases by more than $63 \%$. When the tensile strength reaches $163 \%$, the transmission amplitude then increases by more than $98 \%$. Figure 3D shows the terahertz transmission power spectrum of the MXene/PVAc(latex) structure under different degrees of stretching. When the tensile strength increases, the transmission of the $\mathrm{THz}$ intensity also increases obviously. When the tensile

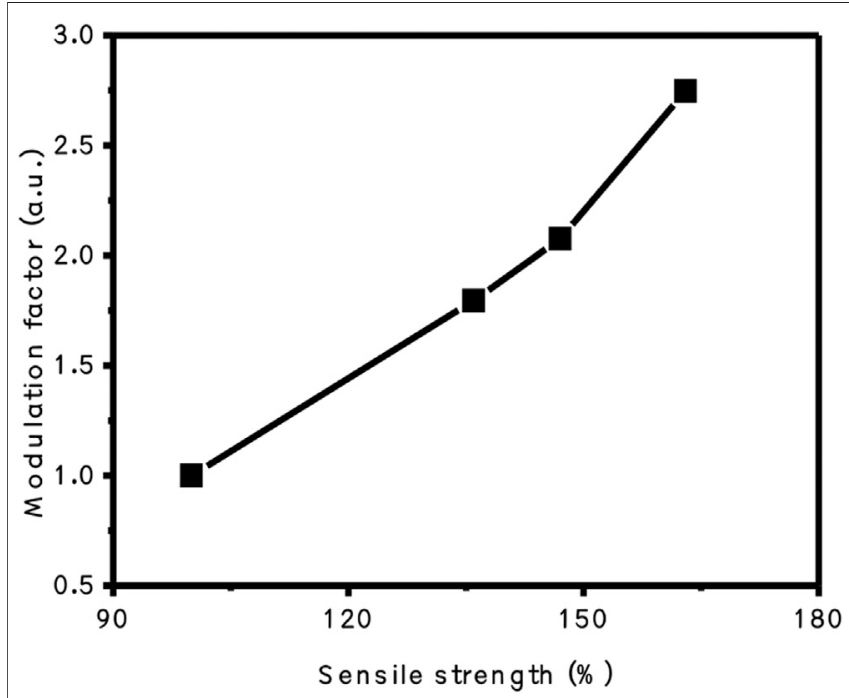

FIGURE 4 | Modulation factor (MF) of samples versus different tensile strengths.

strength reaches $163 \%$, the modulation of the transmission strength is approximately $281 \%$.

To evaluate the modulation performance of the sample, the modulation factor $(M F)$ is introduced and is defined as the change in the integrated terahertz power emission caused by the light excitation intensity: 


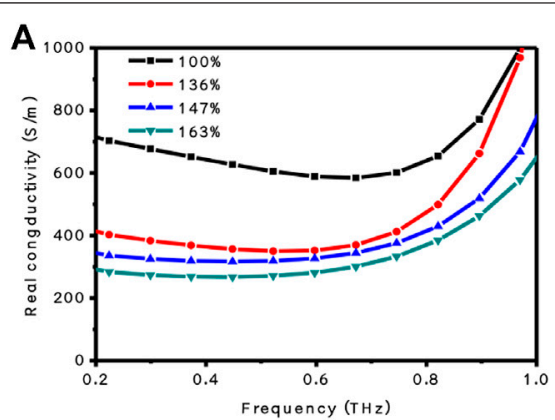

C

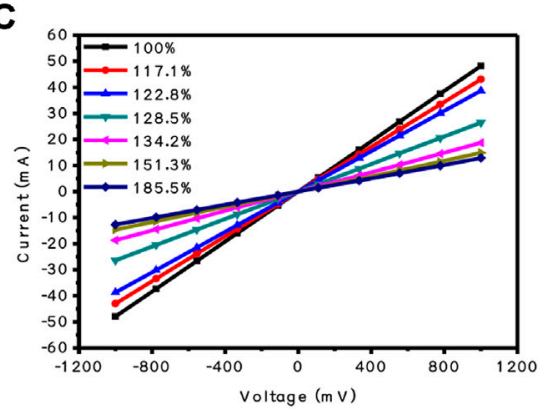

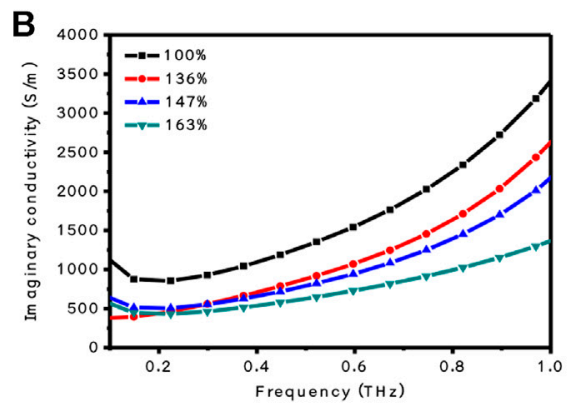

D

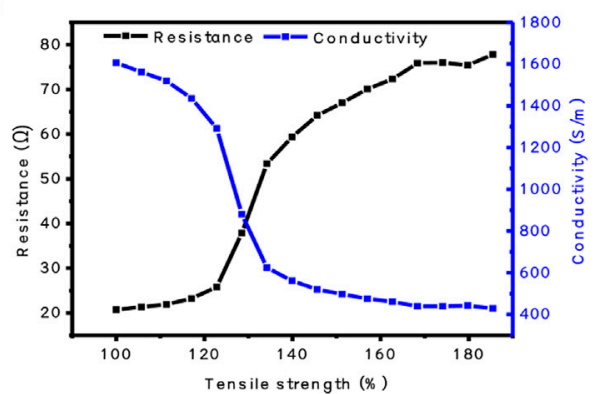

FIGURE 5 | (A) Change in the real part of the conductivity of samples at different tensile degrees. (B) Change in the imaginary part of the conductivity of samples excited by different tensile degrees. (C) I-V diagram for electrical characterization of samples at different tensile degrees. (D) Relationship between the resistance and conductivity of the sample with respect to the tensile degree during electrical characterization.

$$
M F=\frac{\int P_{\text {lax }}(\omega) \mathrm{d} \omega-P_{\text {stretch }}(\omega) \mathrm{d} \omega}{\int P_{\text {lax }}(\omega) \mathrm{d} \omega} .
$$

$P_{\text {lax }}(\omega) \mathrm{d} \omega$ and $P_{\text {stretch }}(\omega) \mathrm{d} \omega$ are the powers of the terahertz signal in the relaxed state and the stretched state, respectively. Figure 4 shows the dependence of the MF on the tensile strength. When the tensile strength was $136 \%$, MF increased by 1.96 . When the tensile strength reaches $147 \%, \mathrm{MF}$ increases to 2.12 . When the tensile strength reaches $163 \%$, MF increases to 2.81 . The change range of MF also increases with the increase of tensile strength. Due to the limitation of material properties, the limit of tensile strength is about $170 \%$. If the tensile degree exceeds this limit, the damage to the substrate cannot be recovered.

To explain the modulation mechanism in further depth, the variations in the conductivity of the samples with the tensile degree have been calculated. From these calculations, we can extract the transient frequency-dependent complex conductivity from the time-domain data of the transmitted $\mathrm{THz}$ pulses, denoted by $E_{\text {strentch }}(\omega)$ and $E_{\text {non-strentch }}(\omega)$. The transmission ratio $T(\omega)$ of the Fourier transform of these two wave forms is related to the complex conductivity as follows [39]:

$$
T_{(\omega)}=\frac{\tilde{E}_{\text {stretch }}(\omega)}{\tilde{E}_{\text {non-stretch }}(\omega)}=\frac{n+1}{n+1+Z_{0} d \tilde{\sigma}(\omega)},
$$

Where $Z_{0}=377 \Omega$ is the impedance of free space, $n=1.5$ is the refractive index of the non-stretch PVAc substrate within the $\mathrm{THz}$ range, and $d$ is the thickness of the MXene coating, which is estimated to be $150 \mu \mathrm{m}$ from the results in Figure 1B. Figures 5A,B show the real part $\sigma_{\mathrm{r}}(\omega)$ and the imaginary part $\sigma_{\mathrm{i}}(\omega)$ of the conductivity of Mxene coating under different tensile degrees, respectively. Because the $\mathrm{THz}$ signal is strongly attenuated by the modulators, the calculation results in the higher and lower frequency ranges are not reliable, and thus we have only presented data measured between 0.1 and $1.0 \mathrm{THz}$ in Figures $\mathbf{5 A , B}$. To make the TDS calculations more scientifically accurate, the electrical experiment was performed as a reference group, with results as shown in Figure 5C. The relationship between the resistance and the degree of stretching is derived from the $I$ - $V$ diagram using the relationship among the current, the voltage and the resistance. The black curve in Figure 5D shows the variation in the resistance of the sample with the degree of drawing, while the blue curve shows the variation in the conductivity of the sample with the degree of drawing. The figure shows that the sample's resistance increases as the tensile strength increases and the sample's conductivity decreases as the tensile strength increases. The increase of the tensile strength from 100 to $136 \%$ is also shown to have a significant effect on the modulation of the tensile strength; at 147 and $163 \%$, the stretching degree has little effect on the modulation. This is consistent with the peak modulation and the $M F$ of the terahertz radiation time-domain spectrum.

\section{CONCLUSION}

A flexible broadband terahertz modulator based on a strain-sensitive MXene/PVAc(latex) material is reported. It is found that the MXene can absorb nearly $80 \%$ of the $\mathrm{THz}$ waves through testing of sponge and latex substrate-based samples. The results obtained show that the transmission rate of the $\mathrm{THz}$ signal increases with increases in the 
degree of stretching. When the stretching degree reaches 136, 147 and $163 \%$, the terahertz time-domain spectrum signals are modulated by 42, 67 and 98\%, respectively. The maximum modulation of the terahertz projection power spectrum reaches $281 \%$. Through an analysis based on $\mathrm{THz}$ TDS and comparison of the electrical properties of the samples, it is concluded that the conductivity of each sample decreases with increasing tensile strength. In addition, the trends for both properties are the same and the order of magnitude remains consistent. The results show that MXene is a type of terahertz modulator that can modulate the terahertz transmission signal intensity of a sample effectively by stretching that sample with a strong force to change the conductivity of the sample.

\section{DATA AVAILABILITY STATEMENT}

The raw data supporting the conclusion of this article will be made available by the authors, without undue reservation.

\section{REFERENCES}

1. Ferguson B, and Zhang X-C (2002). Materials for Terahertz Science and Technology. Nat Mater 1(1):26-33. doi:10.1038/nmat708

2. Tonouchi M (2007). Cutting-edge Terahertz Technology. Nat Photon 1(2): 97-105. doi:10.1038/nphoton.2007.3

3. Padilla WJ, Cich MJ, Azad AK, Averitt RD, Taylor AJ, and Chen HT (2009). A Metamaterial Solid-State Terahertz Phase Modulator. Nat Photon 3(3):148. doi:10.1038/nphoton.2009.3

4. Chen H-T, Padilla WJ, Zide JMO, Gossard AC, Taylor AJ, and Averitt RD (2006). Active Terahertz Metamaterial Devices. Nature 444(7119):597-600. doi: $10.1038 /$ nature 05343

5. Hendry E, Garcia-Vidal FJ, Martin-Moreno L, Rivas JG, Bonn M, Hibbins AP, et al. (2008). Optical Control over Surface-Plasmon-Polariton-Assisted THz Transmission through a Slit Aperture. Phys Rev Lett 100(12):123901. doi:10. 1103/PhysRevLett.100.123901

6. Chen S, Fan F, Miao Y, He X, Zhang K, and Chang S (2016). Ultrasensitive Terahertz Modulation by Silicon-Grown MoS2nanosheets. Nanoscale 8(8): 4713-9. doi:10.1039/c5nr08101g

7. Wen Q-Y, Tian W, Mao Q, Chen Z, Liu W-W, Yang Q-H, et al. (2014). Graphene Based All-Optical Spatial Terahertz Modulator. Sci Rep 4:7409. doi:10.1038/srep07409

8. Mittendorff M, Li S, and Murphy TE. Graphene-based Waveguide-Integrated Terahertz Modulator. ACS Photon (2017). 4(2):316-21. doi:10.1021/ acsphotonics.6b00751

9. Lai W, Huang P, Pelaz B, Pino Pd., and Zhang Q. Enhanced All-Optical Modulation of Terahertz Waves on the Basis of Manganese Ferrite Nanoparticles. J Phys Chem C (2017). 121(39):21634-40. doi:10.1021/acs.jpcc.7b07756

10. Weis P, Garcia-Pomar JL, Höh M, Reinhard B, Brodyanski A, and Rahm M (2012). Spectrally Wide-Band Terahertz Wave Modulator Based on Optically Tuned Graphene. ACS Nano 6(10):9118-24. doi:10.1021/nn303392s

11. Liang GZ, Hu XN, Yu XH, Shen YD, Li LH, Davies AG, et al. (2015). Integrated Terahertz Graphene Modulator with 100\% Modulation Depth. ACS Photon 2(11):1559-66. doi:10.1021/acsphotonics.5b00317

12. Lu M, Li W, and Brown ER (2011). Second-order Bandpass Terahertz Filter Achieved by Multilayer Complementary Metamaterial Structures. Opt Lett 36(7):1071. doi:10.1364/OL.36.001071

13. Harrison H, Lancaster AJI, Konoplev V, Doucas G, Aryshev A, Shevelev M, et al. (2017). The Design of a Non-destructive Single-Shot Longitudinal Bunch Profile Monitor Using Smith-purcell Radiation. Rev Sci Instrum 89:035116. doi:10.18429/JACoW-IPAC2017-MOPAB128

14. Ren L, Pint CL, Booshehri LG, Rice WD, Wang X, Hilton DJ, et al. (2009). Carbon Nanotube Terahertz Polarizer. Nano Lett 9(7):2610-3. doi:10.1021/nl900815s

\section{AUTHOR CONTRIBUTIONS}

YL: Conceptualization, Methodology, Validation, Investigation, Writing-Original Draft; XL: Investigation; TY: Investigation; JL: Investigation; BL: Investigation; JS: Conceptualization, Funding acquisition; BZ: Supervision, Conceptualization, WritingReviewing and Editing, Funding acquisition; FW: Supervision, Conceptualization.

\section{FUNDING}

This research was supported by the National Natural Science Foundation of China (Grant No. 61505125), the Nature Science Foundation of Beijing (Grant No. 4202013), Youth Innovative Research Team of Capital Normal University and High-level Teachers in Beijing Municipal Universities in the Period of 13th Five-year Plan.

15. He X, Zhang Q, Lu G, Ying G, Wu F, and Jiang J (2016). Tunable Ultrasensitive Terahertz Sensor Based on Complementary Graphene Metamaterials. RSC Adv 6(57):52212-8. doi:10.1007/s12200-016-0563-x10.1039/c5ra21974d

16. Grady NK, Heyes JE, Chowdhury DR, Zeng Y, Reiten MT, Azad AK, et al. (2013). Terahertz Metamaterials for Linear Polarization Conversion and Anomalous Refraction. Science 340(6138):1304-7. doi:10.1126/science. 1235399

17. Xiao S, Wang T, Jiang X, Yan X, Cheng L, Wang B, et al. (2017). Strong Interaction Between Graphene Layer and Fano Resonance in Terahertz Metamaterials. J Phys D: Appl Phys 50(9):195101. doi:10.1088/1361-6463/ aa69b1

18. Ji H, Zhang B, Wang W, Lv L, and Shen J (2018). Ultraviolet Light-Induced Terahertz Modulation of an Indium Oxide Film. Opt Express 26(6):7204. doi:10.1364/oe.26.007204

19. Hong JT, Park J-Y, Lee S, and Ahn YH (2016). UV-induced Terahertz Wave Modulation in Free-Standing ZnO Nanowire Films. Opt Mater Express 6(12): 3751. doi:10.1364/OME.6.003751

20. Huang Z, Hao Y, Li Y, Hu H, Wang C, Nomoto A, et al. (2018). Threedimensional Integrated Stretchable Electronics. Nat Electron 1(8):473-80. doi:10.1038/s41928-018-0116-y

21. Mohammed MG, and Kramer R (2017). All-Printed Flexible and Stretchable Electronics. Adv Mater 29(19):1604965. doi:10.1002/adma.201604965

22. Yang W, Feng Y, Si Q, Yan Q, Long P, Dong L, et al. (2019). Efficient Cycling Utilization of Solar-thermal Energy for Thermochromic Displays with Controllable Heat Output. J Mater Chem A 7(1):97-106. doi:10.1039/ C8TA05333B

23. Zhu B, Gong S, and Cheng W (2019). Softening Gold for Elastronics. Chem Soc Rev 48(6):1668-711. doi:10.1039/c8cs00609a

24. Wang Y, Gong S, Wang SJ, Yang X, Ling Y, Yap LW, et al. (2018). Standing Enokitake-like Nanowire Films for Highly Stretchable Elastronics. ACS Nano 12(10):9742-9. doi:10.1021/acsnano.8b05019

25. Xiao D, Zhu M, Sun L, Zhao C, Wang Y, Tong Teo EH, et al. (2019). Flexible Ultra-wideband Terahertz Absorber Based on Vertically Aligned Carbon Nanotubes. ACS Appl Mater Inter 11(46):43671-80. doi:10.1021/acsami. $9 \mathrm{~b} 14428$

26. Suzuki D, Oda S, and Kawano Y (2016). A Flexible and Wearable Terahertz Scanner. Nat Photon 10(12):809-13. doi:10.1038/nphoton.2016.209

27. Cong L, Xu N, Gu J, Singh R, Han J, and Zhang W (2014). Highly Flexible Broadband Terahertz Metamaterial Quarter-Wave Plate. Laser Photon Rev 8(4):626-32. doi:10.1002/lpor.201300205

28. Shi Q, Tian K, Zhu H, Li Z-R, Zhu L-G, Deng H, et al. (2020). Flexible and Giant Terahertz Modulation Based on Ultra-strain-sensitive Conductive Polymer Composites. ACS Appl Mater Inter 12(8):9790-6. doi:10.1021/ acsami.9b21890 
29. Xia Y, Mathis TS, Zhao M-Q, Anasori B, Dang A, Zhou Z, et al. (2018). Thickness-independent Capacitance of Vertically Aligned Liquid-Crystalline MXenes. Nature 557(7705):409-12. doi:10.1038/s41586-018-0109-Z

30. Lukatskaya MR, Kota S, Lin Z, Zhao M-Q, Shpigel N, Levi MD, et al. (2017). Ultra-high-rate Pseudocapacitive Energy Storage in Two-Dimensional Transition Metal Carbides. Nat Energ 2(8):17105. doi:10.1038/nenergy. 2017.105

31. Yan J, Ren CE, Maleski K, Hatter CB, Anasori B, Urbankowski P, et al. (2017). Flexible MXene/Graphene Films for Ultrafast Supercapacitors with Outstanding Volumetric Capacitance. Adv Funct Mater 27(30):1701264. doi:10.1002/adfm.201701264

32. Zhao M-Q, Torelli M, Ren CE, Ghidiu M, Ling Z, Anasori B, et al. (2016). 2D Titanium Carbide and Transition Metal Oxides Hybrid Electrodes for Li-Ion Storage. Nano Energy 30:603-13. doi:10.1016/j.nanoen.2016.10.062

33. Xu Z, Liu G, Ye H, Jin W, and Cui Z (2018). Two-dimensional MXene Incorporated Chitosan Mixed-Matrix Membranes for Efficient Solvent Dehydration. J Membr Sci 563:625-32. doi:10.1016/j.memsci.2018.05.044

34. Ren CE, Hatzell KB, Alhabeb M, Ling Z, Mahmoud KA, and Gogotsi Y (2015). Charge- and Size-Selective Ion Sieving through Ti3C2Tx MXene Membranes. J Phys Chem Lett 6(20):4026-31. doi:10.1021/acs.jpclett.5b01895

35. Muckley ES, Naguib M, Wang H-W, Vlcek L, Osti NC, Sacci RL, et al. (2017). Multimodality of Structural, Electrical, and Gravimetric Responses of Intercalated MXenes to Water. ACS Nano 11(11):11118-26. doi:10.1021/ acsnano.7b05264
36. Choi G, Shahzad F, Bahk Y-M, Jhon YM, Park H, Alhabeb M, et al. (2018). Enhanced Terahertz Shielding of MXenes with Nano-Metamaterials. Adv Opt Mater 6(5):1701076. doi:10.1002/adom.201701076

37. Jhon YI, Koo J, Anasori B, Seo M, Lee JH, Gogotsi Y, et al. (2017). Metallic MXene Saturable Absorber for Femtosecond Mode-Locked Lasers. Adv Mater 29(40):1702496. doi:10.1002/adma.201702496

38. Jhon YI, Seo M, and Jhon YM (2018). First-principles Study of a MXene Terahertz Detector. Nanoscale 10(1):69-75. doi:10.1039/C7NR05351G

39. He T, Zhang B, Wang G-c., Zang M-d., Hou Y-b., and Shen J-l. (2016). High Efficiency THz-Wave Modulators Based on Conjugated Polymer-Based Organic Films. J Phys D: Appl Phys 49(7):075111. doi:10.1088/0022-3727/ 49/7/075111

Conflict of Interest: The authors declare that the research was conducted in the absence of any commercial or financial relationships that could be construed as a potential conflict of interest.

Copyright $\odot 2021 \mathrm{Liu}, \mathrm{Li}$, Yang, Liu, Liu, Shen, Zhang and Wang. This is an openaccess article distributed under the terms of the Creative Commons Attribution License (CC BY). The use, distribution or reproduction in other forums is permitted, provided the original author(s) and the copyright owner(s) are credited and that the original publication in this journal is cited, in accordance with accepted academic practice. No use, distribution or reproduction is permitted which does not comply with these terms. 